\title{
Conservation of the Nrf2-Mediated Gene Regulation of Proteasome Subunits and Glucose Metabolism in Zebrafish
}

\author{
Vu Thanh Nguyen, ${ }^{1,2}$ Yuji Fuse, ${ }^{1,2}$ Junya Tamaoki, ${ }^{1,3}$ Shin-ichi Akiyama, ${ }^{4,5}$ \\ Masafumi Muratani, ${ }^{6}$ Yutaka Tamaru, ${ }^{5}$ and Makoto Kobayashi ${ }^{1}$ \\ ${ }^{1}$ Department of Molecular and Developmental Biology, Faculty of Medicine, University of Tsukuba, Tsukuba 305-8575, Japan \\ ${ }^{2}$ Doctoral Program in Biomedical Sciences, Graduate School of Comprehensive Human Sciences, University of Tsukuba, \\ Tsukuba 305-8575, Japan \\ ${ }^{3}$ College of Biological Sciences, University of Tsukuba, Tsukuba 305-8575, Japan \\ ${ }^{4}$ Division of Nephrology, Department of Internal Medicine, Nagoya University Graduate School of Medicine, Nagoya 466-8550, Japan \\ ${ }^{5}$ Department of Life Sciences, Graduate School of Bioresources, Mie University, Tsu 514-8507, Japan \\ ${ }^{6}$ Department of Genome Biology, Faculty of Medicine, University of Tsukuba, Tsukuba 305-8575, Japan
}

Correspondence should be addressed to Makoto Kobayashi; makobayash@md.tsukuba.ac.jp

Received 26 August 2016; Revised 9 November 2016; Accepted 22 November 2016

Academic Editor: Thomas Kensler

Copyright (C) $2016 \mathrm{Vu}$ Thanh Nguyen et al. This is an open access article distributed under the Creative Commons Attribution License, which permits unrestricted use, distribution, and reproduction in any medium, provided the original work is properly cited.

\begin{abstract}
The Keapl-Nrf2 system is an evolutionarily conserved defense mechanism against oxidative and xenobiotic stress. Besides the exogenous stress response, Nrf2 has been found to regulate numerous cellular functions, including protein turnover and glucose metabolism; however, the evolutionary origins of these functions remain unknown. In the present study, we searched for novel target genes associated with the zebrafish Nrf2 to answer this question. A microarray analysis of zebrafish embryos that overexpressed Nrf2 revealed that 115 candidate genes were targets of Nrf2, including genes encoding proteasome subunits and enzymes involved in glucose metabolism. A real-time quantitative PCR suggested that the expression of 3 proteasome subunits ( $p s m a 3$, psma5, and $p s m b 7$ ) and 2 enzymes involved in glucose metabolism ( $p g d$ and $f b p 1 a$ ) were regulated by zebrafish Nrf2. We thus next examined the upregulation of these genes by an Nrf2 activator, diethyl maleate, using Nrf2 mutant zebrafish larvae. The results of real-time quantitative PCR and whole-mount in situ hybridization showed that all of these 5 genes were upregulated by diethyl maleate treatment in an Nrf2-dependent manner, especially in the liver. These findings implied that the Nrf2-mediated regulation of the proteasome subunits and glucose metabolism is evolutionarily conserved among vertebrates.
\end{abstract}

\section{Introduction}

The Keap1-Nrf2 system is a mechanism that protects cells against xenobiotic and oxidative stress. Under conditions of stress, transcription factor Nrf2 transactivates a wide array of genes, which perform a range of functions, including (but not limited to) the encoding of antioxidant proteins, glutathioneconjugating enzymes, and xenobiotic transporters. This confers an inducible defense against stressors $[1,2]$. Although these genes have been extensively studied as Nrf2 targets, there is increasing evidences to show that Nrf2 activates a wider gene set than was initially considered [3]. Nrf2 regulates the protein turnover by inducing proteasome subunits to confer protective effects against chronic diseases [4] and modifies cellular metabolic processes, for example, the pentose phosphate pathway, which provides NADPH and purine nucleotides that are essential for the redox homeostasis and cellular proliferation $[5,6]$. Because of this multifunctionality, Nrf2 research has become an emerging topic in the medical field $[7,8]$.

The zebrafish has become a popular model vertebrate in basic medical science $[9,10]$. Although it is convenient to use lower vertebrate models for medical research, there is always some concern as to whether the findings are applicable to human medicine. We have investigated the Keap1-Nrf2 system using the zebrafish as a model and revealed that the 
regulation of the system is based on a similar molecular mechanism to mammals [11-15]. Moreover, an analysis of the Nrf2 mutant zebrafish strain, $n r f 2 a^{f h 318}$, revealed that the protective role against oxidative and xenobiotic stress was conserved in the zebrafish [16, 17]. The lineup of the target genes in the zebrafish, which provides the defense function, is also being clarified from recent studies [18]. Our previous study [19] and the study of Hahn et al. [20] reported the performance of microarray analyses using zebrafish larvae treated with potent Nrf2 inducers, diethyl maleate (DEM) and tert-butylhydroquinone (tBHQ), respectively, and found that canonical Nrf2 targets, such as detoxification and antioxidative enzymes were conserved in zebrafish. However, it is still unclear whether the Nrf2-mediated regulation of cellular pathways other than antioxidation and detoxification, for example, protein turnover and glucose metabolism, is conserved among vertebrates or whether it is only present in higher vertebrates.

In the present study, we searched for novel target genes for zebrafish Nrf2 to answer this question. We performed a microarray analysis of zebrafish embryos that overexpressed $\mathrm{Nrf2}$, which was a different approach from previous reports $[19,20]$. The analysis revealed genes encoding proteasome subunits and enzymes involved in glucose metabolism, suggesting that the Nrf2-mediated regulation of both protein turnover and glucose metabolism is evolutionarily conserved among vertebrates.

\section{Materials and Methods}

2.1. Zebrafish and Chemical Treatments. The wild-type (AB) and $n r f 2 a^{f h 318}$ mutant [16] zebrafish strains were used in the present study. The $n r f 2 a^{f h 318}$ strain was maintained by PCRbased genotyping, as described in Fuse et al. [17]. Embryos were obtained by natural mating. For DEM treatment, the larvae were placed in $3 \mathrm{~cm}$ culture dishes at 4 days postfertilization (dpf) and treated with $100 \mu \mathrm{M}$ DEM for $6 \mathrm{~h}$ (Wako, Osaka, Japan). All of the experiments were performed according to methods that were approved by the Animal Experiment Committee of the University of Tsukuba.

2.2. Microarray Analysis. A DNA microarray analysis was performed using custom-made $16 \mathrm{~K}$ MZH chips (GPL14379), as described previously [19]. Biological experiments were carried out in triplicate and microarray analyses were conducted in duplicate or triplicate for each biological experiment. For the overexpression of zebrafish $n r f 2 a$, capped $n r f 2 a$ RNA was synthesized from pCS2nrf2 [11] using an SP6 mMESSAGE mMACHINE in vitro transcription kit (Ambion, Austin, TX). One hundred pg of mRNA was injected into a 1-cell stage embryo by an IM300 microinjector (Narishige, Tokyo, Japan). At $8 \mathrm{~h}$ after injection, the embryos were collected and homogenized with QIAzol reagent (Qiagen, Hilden, Germany) and stored at $-80^{\circ} \mathrm{C}$. A dual-color ratio methodology was applied to compare $n r f 2 a$ RNA-injected embryos with uninjected embryos (Control), according to the manufacturer's protocol for the AceGene DNA microarray (Hitachi Solutions, Tokyo, Japan). Total RNA was extracted according to the manufacturer's instructions for QIAzol reagent (Qiagen), in combination with the clean-up protocol of the RNeasy Mini Kit (Qiagen). Amino-allyl-modified RNA was synthesized using the amino-allyl RNA amplification kit (Sigma-Aldrich, St. Louis, MO) and labeled with monoreactive Cy3 and Cy5 dyes (GE Healthcare, Little Chalfont, UK). The hybridized MZH chips were scanned using the Affymetrix 428 array scanner (Affymetrix, Santa Clara, CA). The microarray data were processed from raw data image files with Affymetrix Jaguar (Affymetrix) and were analyzed using perchip normalization. The processed data were subsequently imported into Excel (Microsoft, Redmond, WA) to compare expression profiles of two samples (injected versus uninjected with nrf2a mRNA). Genes whose expression was affected by the Nrf2a overexpression were selected based on cut-off values of $>1.5$-fold up or $>1.5$-fold down, without considering their significance. A biological pathway analysis was performed using the Reactome database (http://www.reactome.org/). We have deposited the raw data at Gene Expression Omnibus (GEO) under accession number GSE86174, and we can confirm all details are Minimum Information About a Microarray Experiment compliant.

2.3. Real-Time PCR. Total RNA from nrf2a-overexpressing embryos and DEM-treated larvae was prepared according to the procedure that was performed in the microarray analysis. cDNA was synthesized using ReverTra Ace qPCR RT Master Mix (Toyobo, Osaka, Japan) according to the manufacturer's instructions. A quantitative PCR (qPCR) was performed using a 7500 Fast Real-Time PCR System (Thermo Fisher Scientific, Waltham, MA) with THUNDERBIRD SYBR qPCR Mix (Toyobo). The specific primers are listed in Table S1 in Supplementary Material available online at http://dx.doi.org/ $10.1155 / 2016 / 5720574$. The expression level of each gene was normalized to the level of efl $\alpha$.

2.4. In Situ Hybridization Analysis. A whole-mount in situ hybridization analysis was performed as described previously [21]. To construct pKSpsma3, pKSpsma5, pKSpsmb7, pKSpgd, and pKSfbpla, PCR fragments were amplified with primers shown in Table S2 using cDNA synthesized from zebrafish larvae $(4 \mathrm{dpf})$, and ligated with pBluescript II KS+ vector using a DNA Ligation Kit Ver.1 (Takara, Otsu, Japan). Plasmids were linearized by restriction enzymes (pKSpsma3, pKSpsmb7, and pKSpgd: BamHI; pKSpsma5: EcoRI; pKSfbpla: SpeI) and transcribed with T3 RNA polymerase (Roche Diagnostics, Indianapolis, IN) in the presence of DIG RNA labeling mix (Roche Diagnostics) to make RNA probes. The larvae were photographed using an MZ16 microscope (Leica, Wetzlar, Germany) equipped with a DP73 digital camera (Olympus, Tokyo, Japan), followed by PCR-based genotyping.

2.5. Statistical Analysis. The gene expression levels derived from the real-time PCRs were compared using the two-tailed Student's $t$-test. $P$ values of $<0.05$ were considered to indicate statistical significance. 
TABLE 1: The pathways activated in $n r f 2 a$-overexpressing embryos.

\begin{tabular}{|c|c|c|}
\hline$p$ value & Pathway name & Genes \\
\hline $5.27 E-7$ & Detoxification of reactive oxygen species & $t x n, g s t p 1, p r d x 1, g s r, g p x 1 b, p r d x 5, g p x 1 a$ \\
\hline $5.22 E-5$ & Glutathione conjugation & gclc, gsto 2, gstp1, gclm, gsta.1 \\
\hline $6.47 E-4$ & Reduction of cytosolic $\mathrm{Ca}^{++}$levels & atp $2 b 2$, calm $3 a$ \\
\hline $7.86 E-4$ & Apoptosis & lmna, tradd, psma3, psmb7, psma5, tp53bp2a, kpna1, prkcdb \\
\hline $1.04 E-3$ & Programmed cell death & Imna, tradd, psma3, psmb7, psma5, tp53bp2a, kpna1, prkcdb \\
\hline $5.09 E-3$ & Degradation of GLI1 by the proteasome & tpkl, psma3, psmb7, psma5 \\
\hline $5.09 E-3$ & GLI3 is processed to GLI3R by the proteasome & tpkl, psma3, psmb7, psma5 \\
\hline $5.09 E-3$ & Degradation of GLI2 by the proteasome & tpk1, psma3, psmb7, psma5 \\
\hline $5.99 E-3$ & Gluconeogenesis & $p c x b, f b p 1 a, p c k 1, t p k 1$ \\
\hline $7.01 E-3$ & CaM pathway & tpkl, calm3a, prkcdb \\
\hline
\end{tabular}

Bold genes encode proteasome subunits.

\section{Results}

3.1. The Identification of Novel Target Genes for Zebrafish Nrf2 by a Microarray Analysis. To search for the novel target genes of zebrafish Nrf2, we performed a microarray analysis of zebrafish embryos overexpressing Nrf2. In vitro synthesized mRNA of $n r f 2 a$, the functional ortholog of mammalian Nrf2 in zebrafish, was injected into 1-cell stage of zebrafish embryos, and the gene expression in the injected embryos at $8 \mathrm{~h}$ after the injection was examined using $16 \mathrm{KMZH}$ chips, which contain 16,399 probes [19]. In total, 115 genes were found to be upregulated more than 1.5 -fold by the overexpression of $n r f 2 a$ (Table S3).

The lineup of upregulated genes in the microarray of $n r f 2 a$-overexpressing embryos was further analyzed using the Reactome database to determine the biological functions that could be influenced by these upregulated genes (Table 1). Besides antioxidation and glutathione conjugation, proteasome-related pathways were listed, due to the upregulation of the 3 genes encoding the proteasome subunits, psma3, psma5, and psmb7 (proteasome subunits $\alpha 3, \alpha 5$, and $\beta 7$, resp.). It should be noted that none of the genes encoding the proteasome subunits, including these 3 genes, have been identified in previous microarray analyses $[19,20]$.

3.2. Proteasome Subunits Were Regulated by Zebrafish Nrf2. To validate the microarray results, we performed a realtime qPCR. $n r f 2 a$-overexpressing embryos were prepared in the same way as in the microarray analysis. Overexpression of $n r f 2 a$ in mRNA-injected embryos was confirmed by a real-time qPCR (Figure S1, 75.5-fold higher compared to uninjected embryos). As shown in Figure 1(a), psma3 was significantly induced by the overexpression of $n r f 2 a$ (1.51fold). psma5 and psmb7 also tended to be weakly induced by the overexpression of $n r f 2 a$ (1.28- and 1.18-fold, resp.). These results suggest that, similarly to mammals [4], some of the proteasome subunit genes are targets of Nrf2 in the zebrafish.

We then tested whether the expression of 3 proteasome subunit genes is induced by DEM in an Nrf2-dependent manner by a real-time qPCR. Although DEM did not significantly induce any of proteasome subunit genes at $6 \mathrm{~h}$ (Figure 1(b)), psmb7 was induced after $12 \mathrm{~h}$ exposure (Figure 1(c)) in wildtype larvae (1.59-fold) with statistical significance, while the induction in $n r f 2 a^{f h 318 / f h 318}$ mutant (1.33-fold) was weaker than that of wild-type. psma3 and psma5 were also tended to be induced after $12 \mathrm{~h}$ exposure to DEM both in wild-type larvae (1.49- and 1.47-fold, resp.) and in $n r f 2 a^{\text {fh } 318 / f h 318}$ mutant larvae (1.27- and 1.63-fold, resp.).

We speculated that the reason of this unclear Nrf2dependency was due to ubiquitous basal expression of proteasome subunit genes. Thus, we next performed whole-mount in situ hybridization to evaluate tissue-restricted induction of the proteasome subunit genes, since many Nrf2 target genes showed gill-, liver- or nose-specific induction in zebrafish larvae [19]. As we expected, the expression of all three subunit genes was induced in the liver of wild-type and heterozygous mutant $\left(n r f 2 a^{f h 318 /+}\right)$ larvae after $12 \mathrm{~h}$ exposure to DEM (Figure 2). This liver-specific induction was not observed in homozygous mutant siblings $\left(n r f 2 a^{f h 318 / f h 318}\right)$. These results suggest that Nrf2 regulates the gene expression levels of proteasome subunits in zebrafish, especially in the liver.

3.3. Zebrafish Nrf2 Regulates Enzymes That Are Involved in Glucose Metabolism. It is noteworthy that many high-ranked genes in the present microarray analysis were also identified in the previous microarray analyses using DEM- or tBHQtreated larvae (see Table S3, gray highlighted genes) $[19,20]$. This observation indicates that these 27 overlapping genes may be strong candidates for zebrafish Nrf2 targets (Figure 3(a), indicated genes; Table S3, gray highlighted). We therefore analyzed these genes using the Reactome database to search for novel biological pathways that are related to zebrafish Nrf2 (Table 2). As a result, the "Metabolism" pathway was listed within the top 10 categories, in addition to the pathways related to glutathione conjugation and antioxidation. Three catabolic enzymes that are involved in glucose metabolism were included in the "Metabolism" pathway: pckl (phosphoenolpyruvate carboxykinase 1), pcxb (pyruvate carboxylase b), and pgd (phosphogluconate dehydrogenase). Furthermore, looking back on the lineup of genes that were upregulated by the overexpression of $n r f 2 a$ (Table S3), two more related genes were found: taldo1 (transaldolase 1) and fbpla (fructose-1,6-bisphosphatase 1a). 

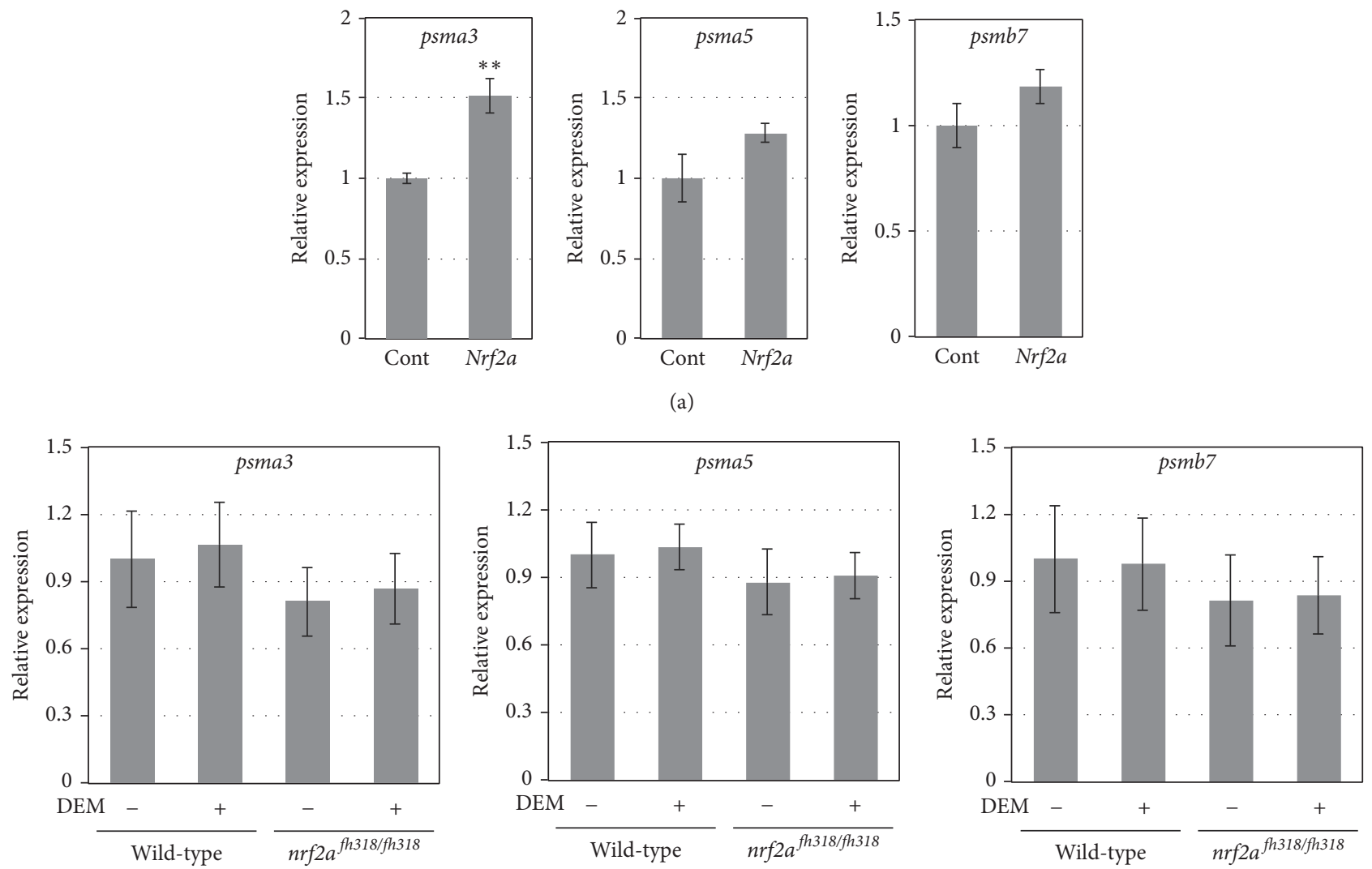

(b)
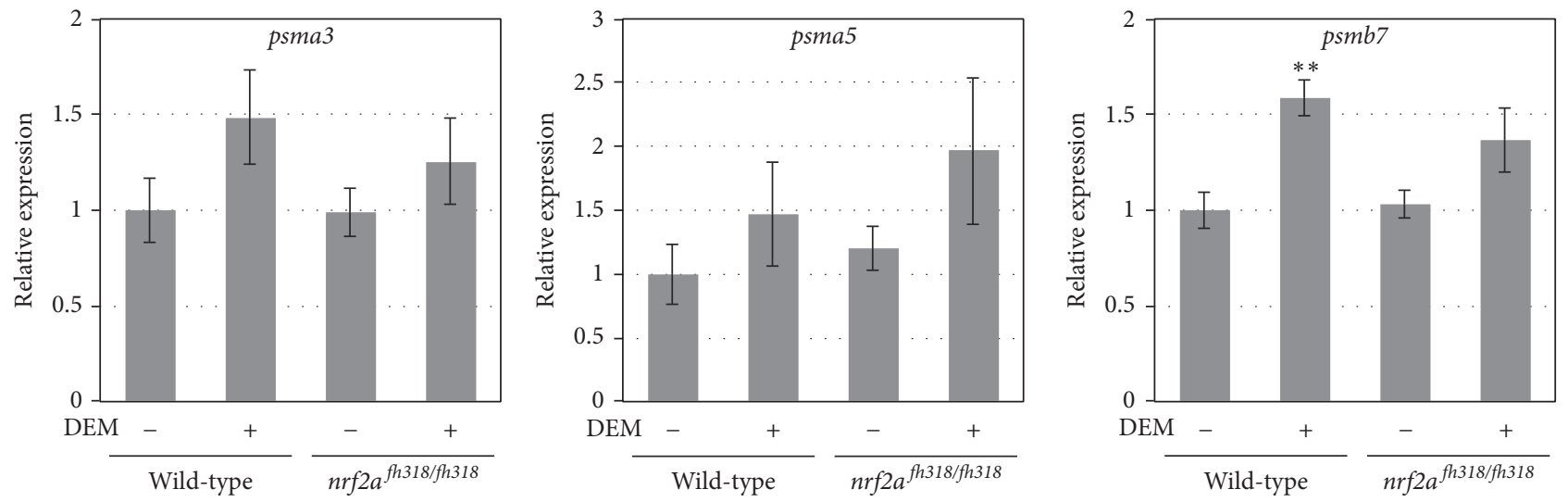

(c)

FIGURE 1: The expression of the proteasome subunit genes. (a) The gene expression of the indicated proteasome subunits in $8 \mathrm{~h}$ postfertilization (hpf) wild-type embryos injected with or without $100 \mathrm{pg}$ of $n r f 2 a$ mRNA at the 1-cell stage was analyzed by a real-time qPCR. Total RNA was extracted from 30 embryos for each sample. The expression of each gene was normalized to that of efl $\alpha$ (means \pm SEM), and the value in uninjected control was set to 1 . Asterisks denote statistical significance (Control versus $n r f 2 a$ overexpression, ${ }^{* *} P<0.01$; Student's $t$-test, $n=6$ for each group). (b) and (c) The gene expression of the indicated proteasome subunits in 4 dpf wild-type or $n r f 2 a^{f h 318 / f h 318}$ mutant larvae that were treated (or not treated) with $100 \mu \mathrm{M}$ DEM for $6 \mathrm{~h} \mathrm{(b)} \mathrm{and} 12 \mathrm{~h} \mathrm{(c)} \mathrm{was} \mathrm{analyzed} \mathrm{by} \mathrm{a} \mathrm{real-time} \mathrm{qPCR.} \mathrm{The} \mathrm{expression} \mathrm{of} \mathrm{each} \mathrm{gene}$ was normalized to that of efl $\alpha$ (means \pm SEM), and the value in untreated wild-type control was set to 1.

To confirm the Nrf2-dependent upregulation of these genes, we next carried out a real-time qPCR using nrf2aoverexpressing embryos. The result indicated that the expression levels of $p g d$ and fbpla (1.40- and 2.76-fold, resp.), but not $p c k 1$, $p c x b$, and taldol (1.21-, 0.75-, and 0.70-fold, resp.), were significantly upregulated by the overexpression of $n r f 2 a$ (Figure 3(b)). For $p g d$ and $f b p l a$, we further analyzed the gene expression in DEM-treated larvae. As shown in Figure 3(c), the DEM treatment induced the expression of both $p g d$ and fbpla in wild-type larvae (5.85- and 2.18-fold, resp.), and 


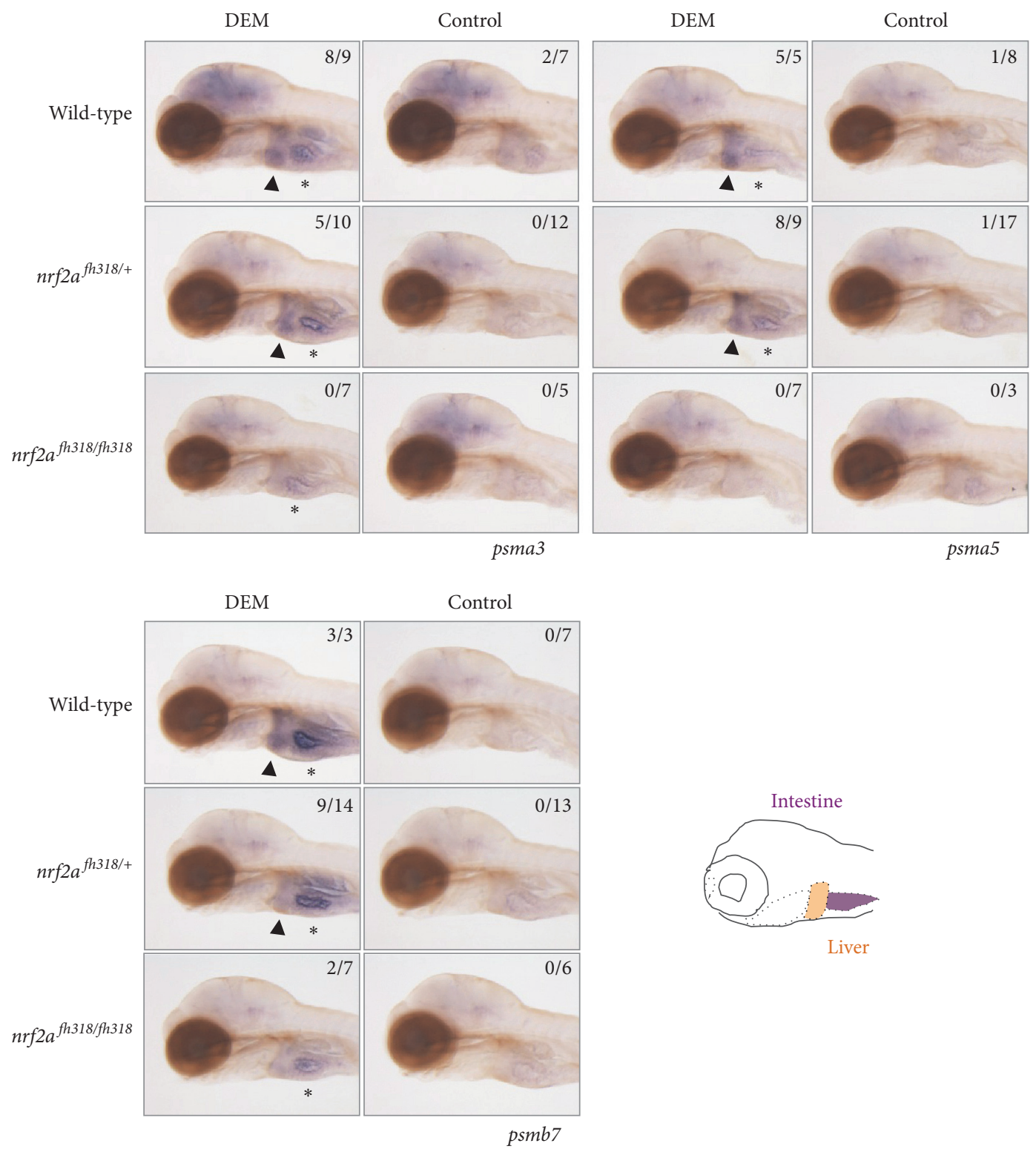

Figure 2: The induction profiles of proteasome subunit genes. Whole-mount in situ hybridization was performed to analyze the induction profiles of psma3, psma5, and psmb7 using $4 \mathrm{dpf} n r f 2 a^{f h 318}$ mutant larvae treated with or without $100 \mu \mathrm{M}$ DEM for $12 \mathrm{~h}$. The arrowheads indicate positive expression in the liver, and asterisks denote the basal expression in the intestine. The numbers in each picture indicate the positive/tested larvae.

the induction was weaker in homozygous $n r f 2 a^{f h 318}$ mutant larvae (2.15- and 1.20-fold, resp.), suggesting clear genetic evidence of Nrf2-dependent regulation.

Induction profiles of these two genes were further analyzed by in situ hybridization (Figure 4). In wild-type and $n r f 2 a^{f h 318}$ heterozygous mutant larvae, pgd was induced specifically in the liver after treatment with DEM for $6 \mathrm{~h}$, while no induction was observed in $n r f 2 a^{f h 318}$ homozygous mutant. fbpla was also induced by DEM in the liver, and this induction was stronger in wild-type and heterozygous mutant compared with homozygous mutant. Weak DEMinduced expression of fbpla was also observed in the gills, but this induction was independent of $n r f 2 a$ genotypes. These results indicate that the Keap1-Nrf2 system, at least in part, regulates the transcription of these enzymes involved in glucose metabolism.

3.4. The Identification of Other Target Genes for Zebrafish Nrf2. The microarray data offered several more candidates for the conserved Nrf2 target gene in zebrafish. We selected 6 genes from the 27 overlapping genes (see Figure 3(a) and Table S3) identified in the current and previous microarray analyses and analyzed their expression using a real-time qPCR. The genes that were selected included $\mathrm{gclm}$ (glutamate-cysteine 


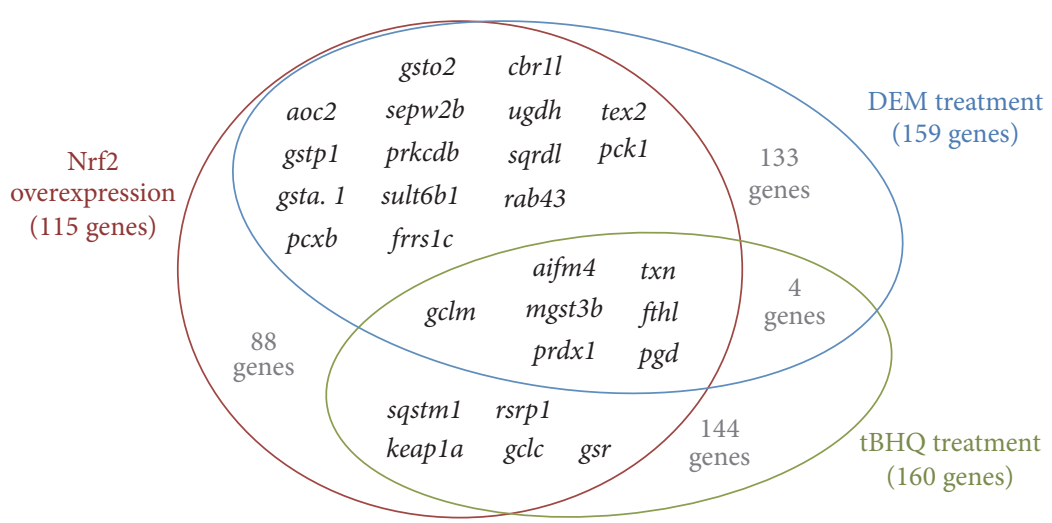

(a)
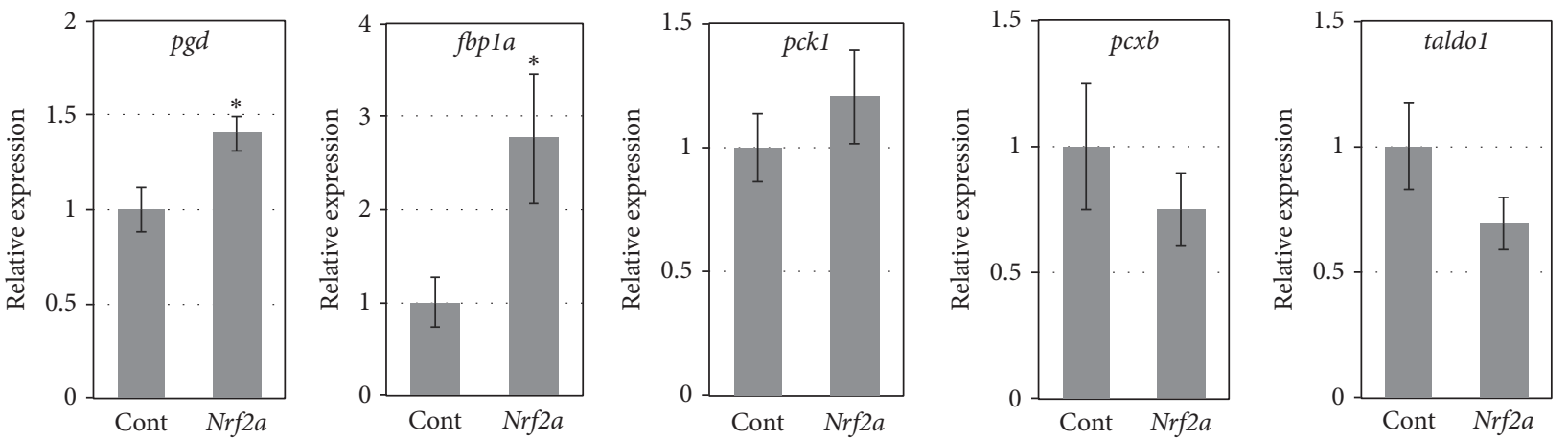

(b)
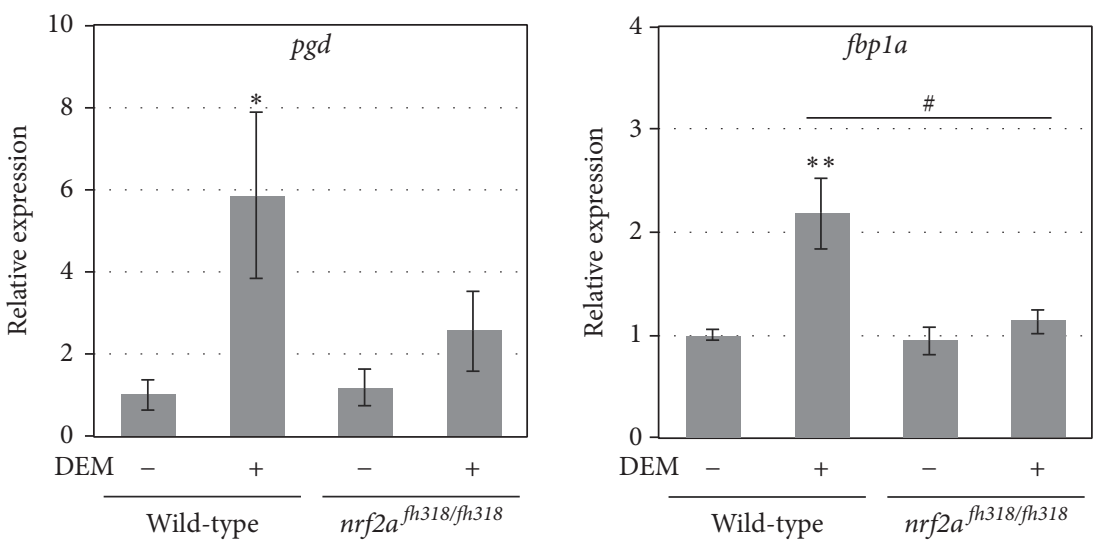

(c)

FIGURE 3: The expression of glucose metabolism-related genes. (a) The upregulated gene lineups from the three microarray experiments were compared. The data of DEM- or tBHQ-treated zebrafish larvae are from Nakajima et al. [19] and Hahn et al. [20], respectively. Numbers in parentheses and in the Venn diagrams denote the numbers of genes which belong to each category. The names of 27 overlapping genes were displayed. (b) The gene expression of the indicated enzymes related to glucose metabolism in 8 hpf wild-type embryos injected with or without $100 \mathrm{pg}$ of $n r f 2 a$ mRNA at the 1-cell stage was analyzed by a real-time qPCR. Asterisks denote statistical significance (Control versus $n r f 2 a$ overexpression, ${ }^{*} P<0.05$; Student's $t$-test, $n=6$ for each group). (c) The gene expression of the indicated enzymes related to glucose metabolism in $4 \mathrm{dpf}$ wild-type or $n r f 2 a^{f h 318 / f h 318}$ mutant larvae treated with or without $100 \mu \mathrm{M}$ DEM for $6 \mathrm{~h}$ was analyzed by a real-time qPCR.

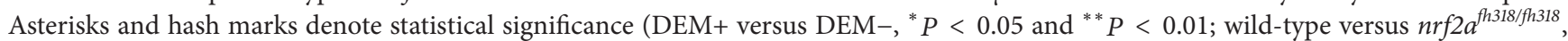
${ }^{\#} P<0.05$; Student's $t$-test, $n=6$ for each group).

ligase, modifier subunit), gsto2 (glutathione $S$-transferase omega 2), gsr (glutathione reductase), sqstm1 (sequestosome 1), and keapla (kelch-like ECH-associated protein la), together with a well-studied nrf2a target, gstp1 (glutathione $S$-transferase pi 1). As shown in Figure 5(a), gclm, gsto2 gsr, and gstp1 were upregulated by the overexpression of $n r f 2 a$ (1.98-, 9.10-, 3.12-, and 6.39-fold, resp.), while sqstm1 and keapla were only slightly induced (1.28- and 1.21-fold, resp.). The expression of these genes was further investigated in the DEM-treated embryos (Figure 5(b)). All of the genes were 
TABLE 2: The pathways activated in both $n r f 2 a$-overexpressing embryos and DEM/tBHQ-treated larvae.

\begin{tabular}{lcc}
\hline$p$ value & Pathway name & Genes \\
\hline $3.33 E-9$ & Glutathione conjugation & gclc, gsto2, gstp1, gclm, gsta.1 \\
$4.13 E-7$ & Phase II conjugation & gclc, gsto2, gstp1, ugdh, gclm, gsta.1 \\
$7.44 E-7$ & Detoxification of reactive oxygen species & txn, gstp1, prdxl, gsr \\
$8.26 E-7$ & Biological oxidations & gclc, gstp1, gsto2, aoc2, ugdh, gclm, gsta.1 \\
$1.39 E-3$ & Vitamin C (ascorbate) metabolism & gsto2 \\
$1.78 E-3$ & Sulfur amino acid metabolism & gclc, sqrdl, gclm \\
$4.77 E-3$ & TP53 regulates metabolic genes & $t x n, p r d x 1, g s r$ \\
$6.76 E-3$ & Glutathione synthesis and recycling & gclc, gclm \\
$8.46 E-3$ & Cellular responses to stress & $t x n, g s t p 1, p r d x 1, g s r$ \\
$8.83 E-3$ & Metabolism & gclc, pckl, gstp1, sqrdl, txn, gsta.1, gsr, pcxb, gsto2, aoc2, pgd, ugdh, gclm \\
\hline
\end{tabular}

Bold genes encode enzymes related to the glucose metabolism process.

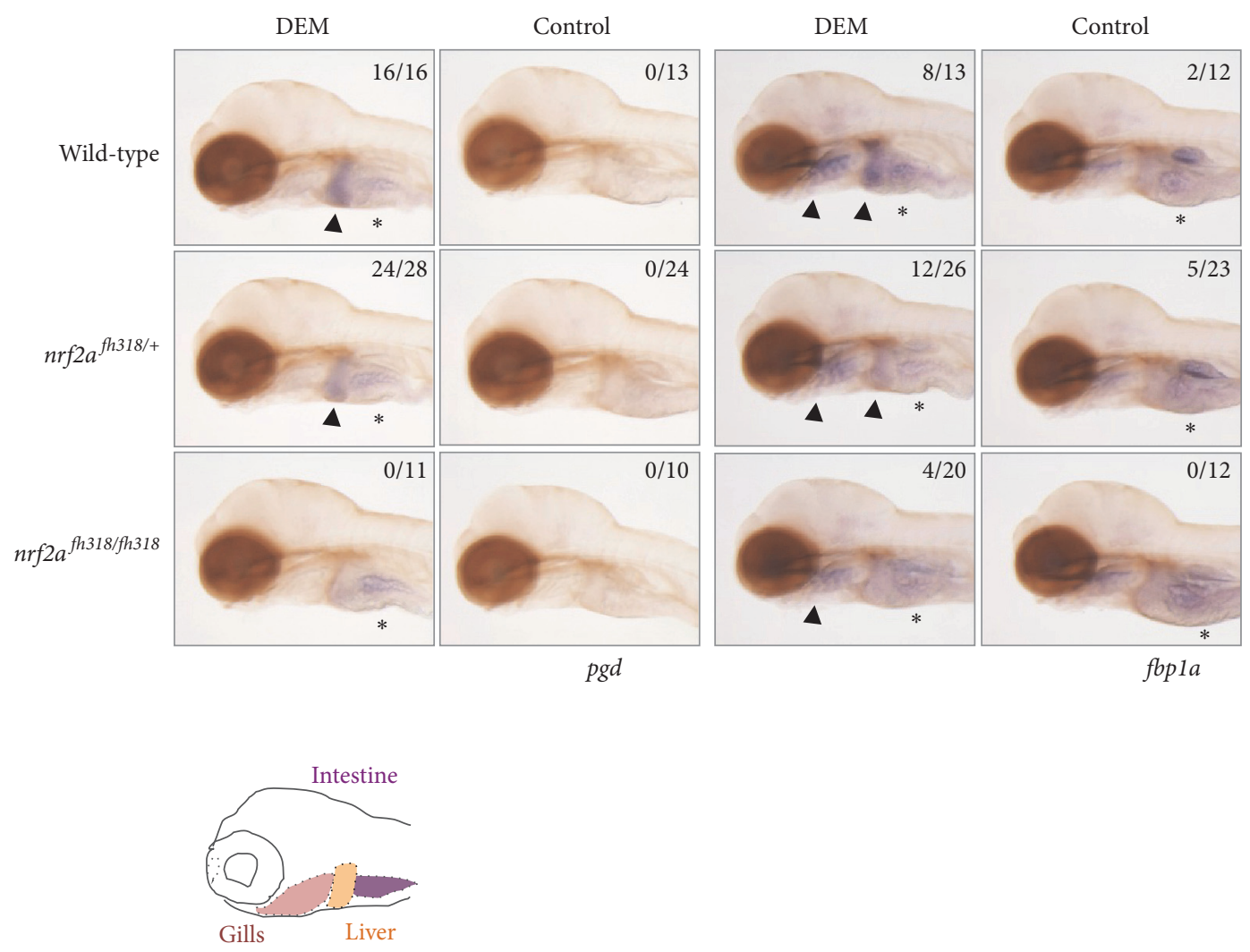

FIgURE 4: The induction profiles of glucose metabolism-related genes. Whole-mount in situ hybridization was performed to analyze the induction profile of $p g d$ and $f b p 1 a$ using $4 \mathrm{pdf} n r f 2 a^{f h 318}$ mutant larvae treated with or without $100 \mu \mathrm{M}$ DEM for 6 h. The arrowheads indicate positive expression in the liver and gills, and asterisks denote the basal expression in the intestine. The numbers in each picture indicate the positive expression in the liver/tested larvae.

strongly induced by DEM in wild-type larvae $(\mathrm{gclm}, 6.37-$ fold; gsto2, 3.99-fold; gsr, 9.40-fold; gstp1, 5.88-fold; sqstm1, 5.44-fold; keapla, 1.72-fold); the induction was weaker, except for keapla, in $n r f 2 a^{f h 318 / f h 318}$ mutant larvae ( $g c l m, 2.80$-fold; gsto2, 2.08-fold; gsr, 6.69-fold; gstp1, 1.94-fold; sqstm1, 2.35fold; keapla, 2.10-fold). The results suggest that these genes are target genes for zebrafish Nrf2.
In our microarray analysis, we also found 55 genes that were downregulated by the overexpression of $n r f 2 a$ (Table S4). None of these genes, except hesxl, was identified in the previous DEM- or tBHQ-based microarray analyses (25 and 82 genes, resp.) $[19,20]$. More importantly, we could not find genes associated with fatty acid metabolism or inflammation-recent studies have implied that these functions are 

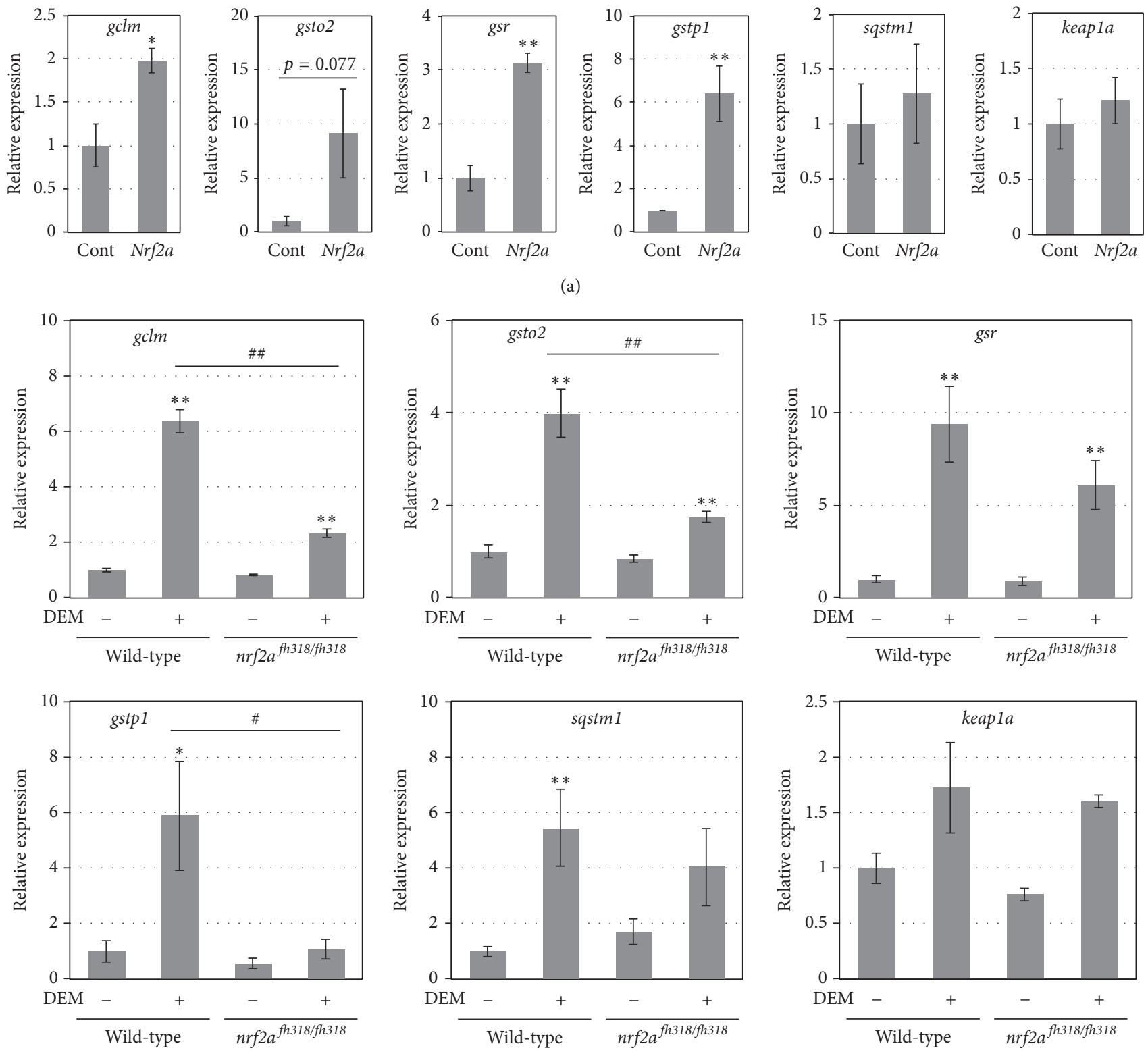

(b)

FIGURE 5: The expression of other candidate genes for zebrafish Nrf2 targets. (a) The expression of the indicated genes in 8 hpf wild-type embryos injected with or without $100 \mathrm{pg}$ of $n r f 2 a$ mRNA at the 1-cell stage was analyzed by a real-time qPCR. Asterisks denote statistical significance (Control versus $n r f 2 a$ overexpression, ${ }^{*} P<0.05$ and ${ }^{* *} P<0.01$; Student's $t$-test, $n=6$ for each group). (b) The expression of the indicated genes in $4 \mathrm{dpf}$ wild-type or $n r f 2 a^{f h 318 / f h 318}$ mutant larvae treated with or without $100 \mu \mathrm{M}$ DEM for $6 \mathrm{~h}$ was analyzed by a realtime qPCR. Asterisks and hash marks denote statistical significance (DEM+ versus DEM-, ${ }^{*} P<0.05$ and ${ }^{* *} P<0.01$; wild-type versus

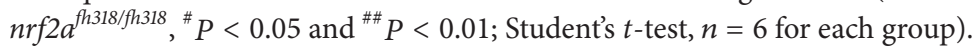

negatively regulated by $\mathrm{Nrf2}[5,22,23]$. The evolutional conservation related to the Nrf2-dependent negative regulation of some types of genes remains unclear.

\section{Discussion}

In the present study, we found that the three proteasome subunits in the $20 \mathrm{~S}$ core particle were regulated by zebrafish Nrf2, one of which ( $p s m b 7)$ has a trypsin-like protease activity; the others (psma3 and psma5) comprise the $\alpha$ ring structure [24]. In mammals, multiple subunits of proteasome have also been shown to be regulated by $\mathrm{Nrf2}$ at the transcriptional level [4], suggesting the presence of a conserved regulatory mechanism among vertebrates. Consistent with the report by Kwak et al. [4], which showed the transcriptional induction of proteasome subunits in mice liver after treatment with D3T, an Nrf2-activating antioxidant, induction was observed dominantly in the liver of zebrafish larvae. Although most Nrf2 
target genes tested in zebrafish larvae showed the induction in the nose and gills in addition to the liver [19], proteasome subunit genes were induced only in the liver. The molecular basis of this liver specificity together with their slow induction should be characterized in the future. As the Nrf2dependent upregulation of the proteasome function is suggested to be important in the defense against oxidative stress, endoplasmic reticulum stress, and senescence [25-27], it is anticipated that the regulatory context should be clarified for medical applications. The zebrafish model can also provide a good model for further study.

Enzymes related to glucose metabolism were also targeted by Nrf2 in zebrafish. Phosphogluconate dehydrogenase (Pgd) is an enzyme that converts 6-phosphogluconate to ribulose 5phosphate in the oxidative branch of the pentose phosphate pathway, which produces NADPH as well as purine nucleotides [28]. The Nrf2-dependent upregulation of $p g d$ has also been demonstrated in mice [6]. The upregulation of $p g d$ may confer protection against stressors by augmenting the supply of NADPH, an essential cofactor for redox homeostasis [5], and the activation of nucleotide biosynthesis through the pentose phosphate pathway, which may lead to the metabolic remodeling in cancer cells [6]. The zebrafish has recently emerged as an important model in cancer biology $[29,30]$. It will be worthwhile to study the Nrf2-dependent metabolic remodeling that takes place in carcinogenesis using zebrafish. In addition to $p g d$, the Nrf2-depedent transcriptional regulation of fructose-1,6-bisphosphatase ( $\mathrm{Fbp}$ ), an enzyme that converts fructose 1,6-bisphosphate to fructose 6-phosphate in gluconeogenesis, was observed specifically in the liver. Further study on the mechanism underlying this liverspecific induction will provide an important clue to understand the biological significance of Nrf2-dependent regulation of glucose metabolism.

In the present microarray analysis, the cut-off value for significant upregulation by the overexpression of $n r f 2 a$ was set at a 1.5 -fold change instead of a 2 -fold change in the previous studies $[19,20]$. It was effective, since 4 out of 6 genes, as we validated the microarray data by a real-time qPCR with statistical significance, showed less than 2-fold change in the microarray analysis (see Figure S2 and Table S3). A correlation in the fold changes between microarray and real-time qPCR data was relatively poor; thus, it is worthwhile to pick up not only high-ranked genes in the microarray analysis but also low-ranked genes for further analyses.

The unique point of the present study is that genes were screened using $n r f 2 a$-overexpressing embryos. A major approach for the microarray-based screening of Nrf2-target genes is the use of cells/tissues/animals treated with Nrf2activating compounds [31-33] as is the case for the previous screen studies that used zebrafish $[19,20]$. Another approach is the use of Keap1 knockout cells/tissues [5, 34]. Since Keap1 is an E3 ubiquitin ligase that targets Nrf2, the disruption of its gene increases the stability of Nrf2 and leads to the constitutive activation of Nrf2. A study by Yates et al. [22] which compared sets of genes upregulated by treatment with a potent Nrf2-activating compound (CDDO-Im) with those upregulated by liver-specific Keapl knockout is of particular interest. Keap1-knockout activated Nrf2-dependent transcription more strongly than the pharmacological activation; as a result greater numbers of Nrf2-regulated genes were detected [22]. It is possible that similar effects were observed in the overexpression method of the present study.

\section{Conclusions}

In conclusion, we found novel Nrf2-target genes in zebrafish by a microarray analysis using $n r f 2 a$-overexpressing zebrafish embryos. The basal expression levels of proteasome subunits were revealed to be regulated by zebrafish Nrf2. In addition, enzymes involved in the pentose phosphate pathway and gluconeogenesis were found to be under Nrf2-dependent transcriptional control. These results suggest that the Nrf2mediated regulation of the genes related to protein turnover and glucose metabolism, at least a part of them, was evolutionarily conserved in vertebrates.

\section{Competing Interests}

The authors declare that they have no competing interests.

\section{Authors' Contributions}

Vu Thanh Nguyen and Yuji Fuse contributed equally to this work.

\section{Acknowledgments}

The authors thank Masayuki Yamamoto for valuable suggestions and Hiroshi Kaneko, Li Li, Yaeko Nakajima-Takagi, Tadayuki Tsujita, and Takeshi Wakasa for experimental help. This work was supported by Grants-in-Aids from the Ministry of Education, Science, Sports and Culture of Japan (to Makoto Kobayashi) (21659043, 24590340, 25118705, 26116705, and 26520101) and the grant from the Yamazaki Spice Promotion Foundation.

\section{References}

[1] T. Suzuki and M. Yamamoto, "Molecular basis of the Keap1Nrf2 system," Free Radical Biology and Medicine, vol. 88, pp. 93100, 2015.

[2] M. Kobayashi and M. Yamamoto, "Nrf2-Keap1 regulation of cellular defense mechanisms against electrophiles and reactive oxygen species," Advances in Enzyme Regulation, vol. 46, no. 1, pp. 113-140, 2006.

[3] J. D. Hayes and A. T. Dinkova-Kostova, "The Nrf2 regulatory network provides an interface between redox and intermediary metabolism," Trends in Biochemical Sciences, vol. 39, no. 4, pp. 199-218, 2014.

[4] M.-K. Kwak, N. Wakabayashi, J. L. Greenlaw, M. Yamamoto, and T. W. Kensler, "Antioxidants enhance mammalian proteasome expression through the Keap1-Nrf2 signaling pathway," Molecular and Cellular Biology, vol. 23, no. 23, pp. 8786-8794, 2003.

[5] K. C. Wu, J. Y. Cui, and C. D. Klaassen, "Beneficial role of Nrf2 in regulating NADPH generation and consumption," Toxicological Sciences, vol. 123, no. 2, pp. 590-600, 2011. 
[6] Y. Mitsuishi, K. Taguchi, Y. Kawatani et al., "Nrf2 redirects glucose and glutamine into anabolic pathways in metabolic reprogramming," Cancer Cell, vol. 22, no. 1, pp. 66-79, 2012.

[7] D. V. Chartoumpekis, N. Wakabayashi, and T. W. Kensler, "Keap1/Nrf2 pathway in the frontiers of cancer and non-cancer cell metabolism," Biochemical Society Transactions, vol. 43, pp. 639-644, 2015.

[8] T. Suzuki, H. Motohashi, and M. Yamamoto, "Toward clinical application of the Keap1-Nrf2 pathway," Trends in Pharmacological Sciences, vol. 34, no. 6, pp. 340-346, 2013.

[9] C. A. MacRae and R. T. Peterson, "Zebrafish as tools for drug discovery," Nature Reviews Drug Discovery, vol. 14, no. 10, pp. 721-731, 2015.

[10] J. B. Phillips and M. Westerfield, "Zebrafish models in translational research: tipping the scales toward advancements in human health," Disease Models and Mechanisms, vol. 7, no. 7, pp. 739-743, 2014.

[11] M. Kobayashi, K. Itoh, T. Suzuki et al., "Identification of the interactive interface and phylogenic conservation of the Nrf2Keap1 system," Genes to Cells, vol. 7, no. 8, pp. 807-820, 2002.

[12] Y. Takagi, M. Kobayashi, L. Li, T. Suzuki, K. Nishikawa, and M. Yamamoto, "MafT, a new member of the small Maf protein family in zebrafish," Biochemical and Biophysical Research Communications, vol. 320, no. 1, pp. 62-69, 2004.

[13] T. Suzuki, Y. Takagi, H. Osanai et al., "Pi-class glutathione Stransferase genes are regulated by Nrf2 through an evolutionarily conserved regulatory element in zebrafish," Biochemical Journal, vol. 388, no. 1, pp. 65-73, 2005.

[14] L. Li, M. Kobayashi, H. Kaneko, Y. Nakajima-Takagi, Y. Nakayama, and M. Yamamoto, "Molecular evolution of Keap1: two Keap1 molecules with distinctive intervening region structures are conserved among fish," Journal of Biological Chemistry, vol. 283, no. 6, pp. 3248-3255, 2008.

[15] M. Kobayashi, L. Li, N. Iwamoto et al., "The antioxidant defense system Keap1-Nrf2 comprises a multiple sensing mechanism for responding to a wide range of chemical compounds," Molecular and Cellular Biology, vol. 29, no. 2, pp. 493-502, 2009.

[16] K. Mukaigasa, L. T. P. Nguyen, L. Li, H. Nakajima, M. Yamamoto, and M. Kobayashi, "Genetic evidence of an evolutionarily conserved role for $\mathrm{Nrf} 2$ in the protection against oxidative stress," Molecular and Cellular Biology, vol. 32, no. 21, pp. 44554461, 2012.

[17] Y. Fuse, V. T. Nguyen, and M. Kobayashi, "Nrf2-dependent protection against acute sodium arsenite toxicity in zebrafish," Toxicology and Applied Pharmacology, vol. 305, pp. 136-142, 2016.

[18] M. E. Hahn, A. R. Timme-Laragy, S. I. Karchner, and J. J. Stegeman, "Nrf2 and Nrf2-related proteins in development and developmental toxicity: insights from studies in zebrafish (Danio rerio)," Free Radical Biology and Medicine, vol. 88, pp. 275-289, 2015.

[19] H. Nakajima, Y. Nakajima-Takagi, T. Tsujita et al., "Tissue-restricted expression of Nrf2 and its target genes in zebrafish with gene-specific variations in the induction profiles," PLOS ONE, vol. 6, no. 10, Article ID e26884, 2011.

[20] M. E. Hahn, A. G. McArthur, S. I. Karchner et al., "The transcriptional response to oxidative stress during vertebrate development: effects of tert-butylhydroquinone and 2,3,7,8-tetrachlorodibenzo-p-dioxin," PLoS ONE, vol. 9, no. 11, Article ID e113158, 2014.
[21] Y. Fuse, H. Nakajima, Y. Nakajima-Takagi, O. Nakajima, and M. Kobayashi, "Heme-mediated inhibition of Bachl regulates the liver specificity and transience of the Nrf2-dependent induction of zebrafish heme oxygenase 1," Genes to Cells, vol. 20, no. 7, pp. 590-600, 2015.

[22] M. S. Yates, Q. T. Tran, P. M. Dolan et al., "Genetic versus chemoprotective activation of Nrf2 signaling: overlapping yet distinct gene expression profiles between Keapl knockout and triterpenoid-treated mice," Carcinogenesis, vol. 30, no. 6, pp. 10241031, 2009.

[23] E. H. Kobayashi, T. Suzuki, R. Funayama et al., "Nrf2 suppresses macrophage inflammatory response by blocking proinflammatory cytokine transcription," Nature Communications, vol. 7, Article ID 11624, 2016.

[24] R. J. Tomko Jr. and M. Hochstrasser, "Molecular architecture and assembly of the eukaryotic proteasome," Annual Review of Biochemistry, vol. 82, pp. 415-445, 2013.

[25] A. M. Pickering, R. A. Linder, H. Zhang, H. J. Forman, and K. J. A. Davies, "Nrf2-dependent induction of proteasome and $\mathrm{Pa} 28 \alpha \beta$ regulator are required for adaptation to oxidative stress," The Journal of Biological Chemistry, vol. 287, no. 13, pp. 10021-10031, 2012.

[26] S. Lee, E.-G. Hur, I.-G. Ryoo, K.-A. Jung, J. Kwak, and M.-K. Kwak, "Involvement of the Nrf2-proteasome pathway in the endoplasmic reticulum stress response in pancreatic $\beta$-cells," Toxicology and Applied Pharmacology, vol. 264, no. 3, pp. 431438, 2012.

[27] S. Kapeta, N. Chondrogianni, and E. S. Gonos, "Nuclear erythroid factor 2-mediated proteasome activation delays senescence in human fibroblasts," Journal of Biological Chemistry, vol. 285, no. 11, pp. 8171-8184, 2010.

[28] A. Stincone, A. Prigione, T. Cramer et al., "The return of metabolism: biochemistry and physiology of the pentose phosphate pathway," Biological Reviews, vol. 90, no. 3, pp. 927-963, 2015.

[29] R. M. White, "Cross-species oncogenomics using zebrafish models of cancer," Current Opinion in Genetics and Development, vol. 30, pp. 73-79, 2015.

[30] J. Yen, R. M. White, and D. L. Stemple, "Zebrafish models of cancer: progress and future challenges," Current Opinion in Genetics and Development, vol. 24, no. 1, pp. 38-45, 2014.

[31] R. K. Thimmulappa, K. H. Mai, S. Srisuma, T. W. Kensler, M. Yamamoto, and S. Biswal, "Identification of Nrf2-regulated genes induced by the chemopreventive agent sulforaphane by oligonucleotide microarray," Cancer Research, vol. 62, no. 18, pp. 5196-5203, 2002.

[32] M.-K. Kwak, N. Wakabayashi, K. Itoh, H. Motohashi, M. Yamamoto, and T. W. Kensler, "Modulation of gene expression by cancer chemopreventive dithiolethiones through the Keap1Nrf2 pathway. Identification of novel gene clusters for cell survival," The Journal of Biological Chemistry, vol. 278, no. 10, pp. 8135-8145, 2003.

[33] R. Hu, C. Xu, G. Shen et al., "Identification of Nrf2-regulated genes induced by chemopreventive isothiocyanate PEITC by oligonucleotide microarray," Life Sciences, vol. 79, no. 20, pp. 1944-1955, 2006.

[34] H. Okawa, H. Motohashi, A. Kobayashi, H. Aburatani, T. W. Kensler, and M. Yamamoto, "Hepatocyte-specific deletion of the keap1 gene activates Nrf2 and confers potent resistance against acute drug toxicity," Biochemical and Biophysical Research Communications, vol. 339, no. 1, pp. 79-88, 2006. 


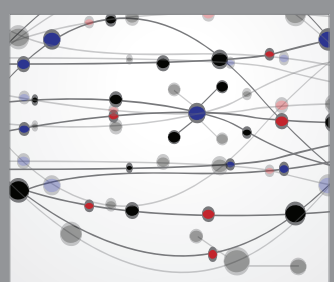

The Scientific World Journal
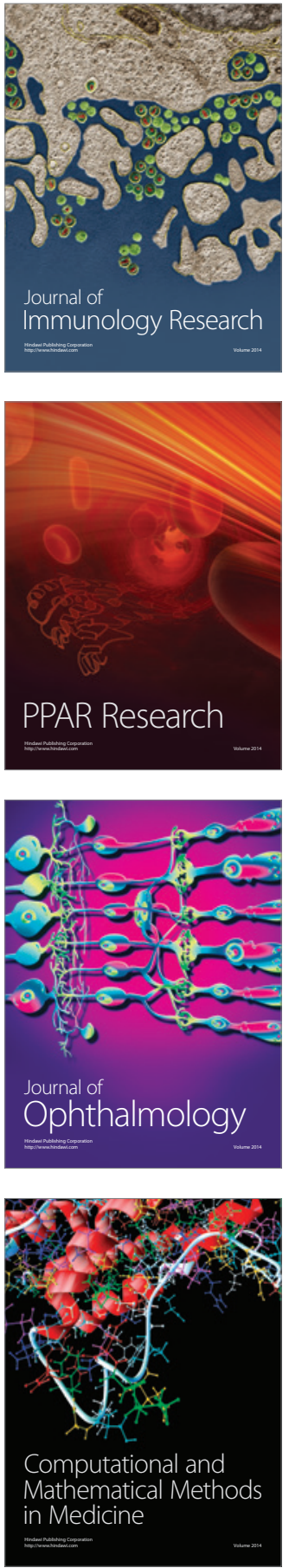

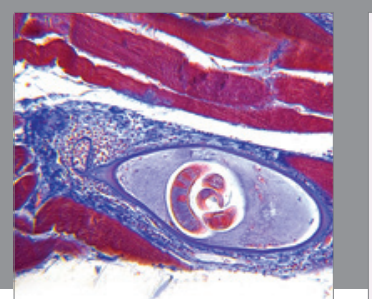

Gastroenterology Research and Practice

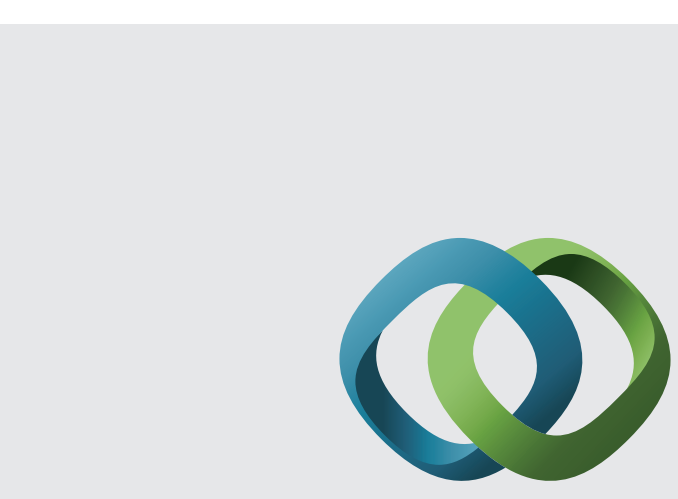

\section{Hindawi}

Submit your manuscripts at

http://www.hindawi.com
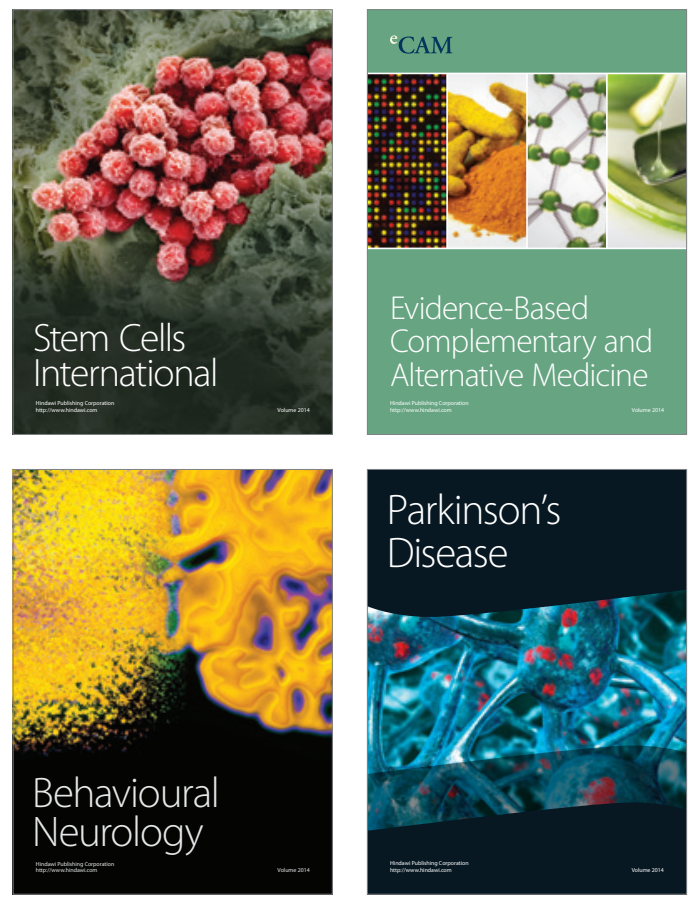
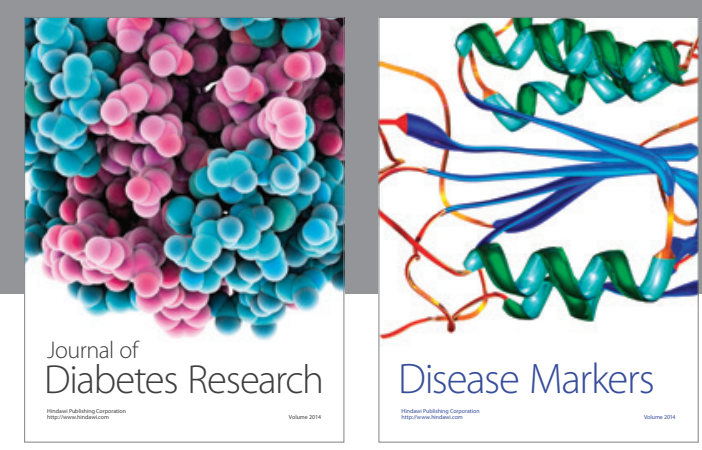

Disease Markers
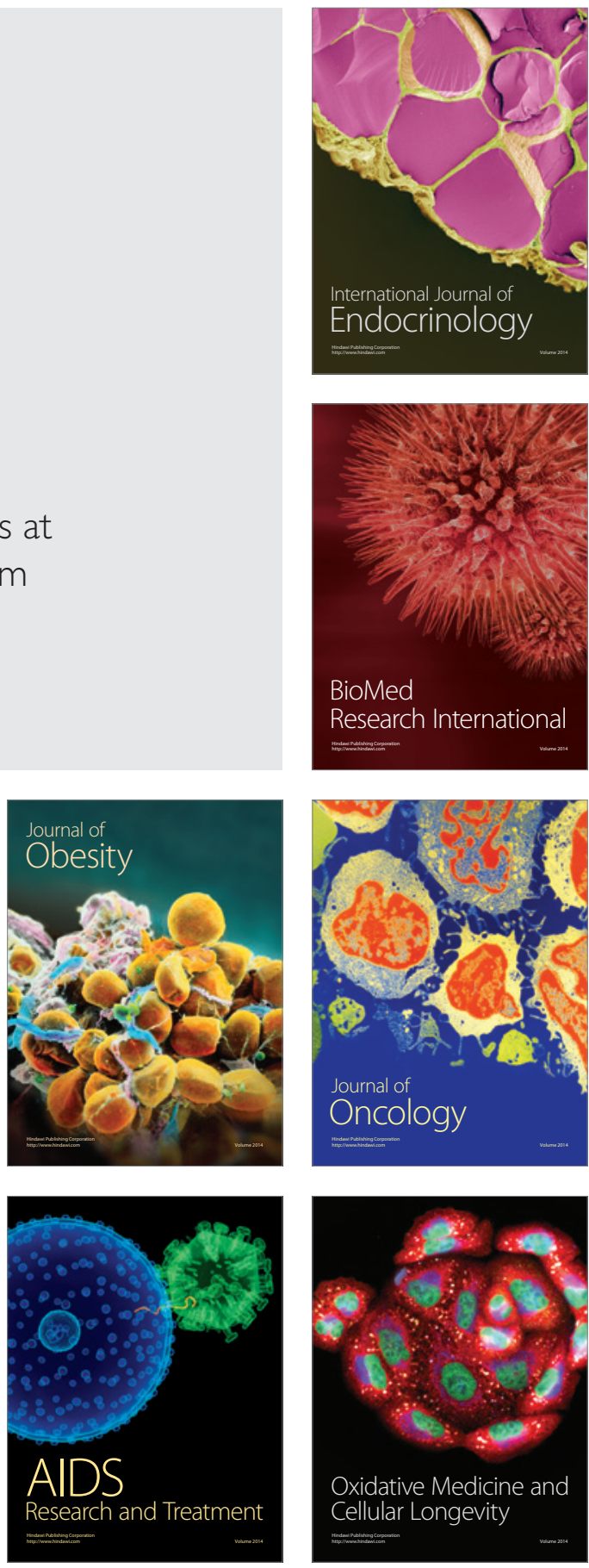